\title{
UNCERTAINTY PRINCIPLES FOR ORTHONORMAL SEQUENCES RELATED TO LAGUERRE HYPERGROUP
}

\author{
SElma NegZaoui
}

Abstract. In this paper, we deal with Laguerre hypergroup $\mathbb{K}=[0,+\infty) \times \mathbb{R}$. We prove an analogous of a time-frequency localization theorem for orthonormal sequences in $L^{2}(\mathbb{K})$. As consequence we obtain an analogous of Shapiro's Umbrella theorem. Also, we provide a mean dispersion inequality. Finally, we get a strong version of the uncertainty inequality for orthonormal bases of $L^{2}(\mathbb{K})$.

Mathematics subject classification (2010): 42B10, 42C25.

Keywords and phrases: Uncertainty inequality, Laguerre hypergroup, time-frequency localization theorem, mean dispersion inequality.

\section{REFERENCES}

[1] M. Assal \& H. Ben Abdallah, Generalized Besov type spaces on the Laguerre hypergroup, AnnalesMathematiques Blaise Pascal 12, (2005), 117-145.

[2] M. Cowling \& J. F. PRICE, Generalizations of Heisenberg's inequality, Lecture Notes in Math. 992, (1983), 443-449.

[3] D. L. Donoho \& P. B. STARK, Uncertainty principles and signal recovery, SIAM J. Appl. Math. 49 (3), (1989), 906-931.

[4] L. Gallardo \& K. TRIMÈche, An $L^{p}$-version of Hardy's theorem for the Dunkl transform, J. Aust. Math. Soc. 77, Part 3, (2004), 371-382.

[5] S. Ghobber And PH. Jaming, Strong annihilating pairs for the Fourier-Bessel transform, J. Math. Anal. Appl. 377, (2011), 501-515.

[6] S. Ghobber \& P. Jaming, Uncertainty principles for integral operators, Stud. Math. 220, No. 3, (2014), 197-220.

[7] S. GuliYev, Polar coordinates in Laguerre hypergroup, Khazar J. Math. 2 (2006), 11-20.

[8] G. H. HARDY, A theorem concerning Fourier transforms, J. Lond. Math. Soc. 8, (1933), 227-231.

[9] W. HEISENBERG, $\ddot{U}$ ber den anschaulichen inhalt der quantentheoretischen kinematik und machanik, Z. f. Physik 43, (1927), 172-198.

[10] P. JAMING, Uncertainty principles for orthonormal bases, Sémin. Équ. Dériv. Partielles, Ec. Polytech., XV, (2006).

[11] P. JAming \& A. M. Powell, Uncertainty principles for orthonormal sequences, J. Funct. Anal. 243, (2007), 611-630.

[12] E. Malinnikova, Orthonormal Sequences in $L^{2}\left(R^{d}\right)$ and Time Frequency Localization, J. Fourier Anal. Appl. 16, (2010), 983-1006.

[13] Y. Meyer, Principe d'incertitude bases hilbertiennes et algèbres d'opérateurs, Sémin. Bourbaki, 662, (1985-1986), 209-223.

[14] G. W. Morgan, A note on Fourier transforms, J. London Math. Soc., 9, (1934), 188-192.

[15] M. M. NeSsibI \& K. TRIMÈCHE, Inversion of the Radon Transform on the Laguerre hypergroup by using generalized wavelets, J. Math. Anal. Appl. 208 (1997), 337-363.

[16] A. M. PowelL, Time-frequency mean and variance sequences of orthonormal bases, J. Fourier Anal. Appl. 11 (4), (2005), 375-387.

[17] A. Rahmouni, Uncertainty Inequalities on Laguerre Hypergroup, Mediterr. J. Math. 10 (2013), 333351. 
[18] H. S. SHAPIRO, Uncertainty principles for bases in L2(R), unpublished manuscript.

[19] K. STEMPAK, Mean summability methods for Laguerre series, Trans. AMS. 322 (2) (1990), 129-147. 